\title{
Sex-on-Premise Venues, Associated Risk Behaviors, and Attitudes toward Venue-Based HIV Testing among Men Who Have Sex with Men in Lima, Perú
}

Alexander Lankowski ( $\sim$ alexlankowski@gmail.com )

University of Pennsylvania https://orcid.org/0000-0002-4769-7242

Hugo Sánchez

Epicentro Peru

José Hidalgo

Via Libre

Robinson Cabello

Via Libre

Ann Duerr

Fred Hutchinson Cancer Research Center

Research article

Keywords: HIV; sexual behavior; MSM; Perú; sex-on-premise venue; sauna; HIV testing; HIV prevention; sexually transmitted infection

Posted Date: February 21st, 2020

DOI: https://doi.org/10.21203/rs.2.18416/v2

License: (c) (i) This work is licensed under a Creative Commons Attribution 4.0 International License.

Read Full License

Version of Record: A version of this preprint was published at BMC Public Health on April 19th, 2020. See the published version at https://doi.org/10.1186/s12889-020-08604-w. 


\section{Abstract}

Background. In Perú, HIV disproportionately affects men who have sex with men (MSM). Despite widespread access to treatment, the high rate of new HIV infections has remained unchanged over the last decade. Low knowledge of HIV status associated with late diagnosis is a key factor underlying the high HIV incidence observed in this setting, creating conditions for efficient onward transmission. Improving access to HIV testing and prevention services for those at highest risk is an important public health priority. Sex-on-premise venues (SOPVs) - saunas, sex clubs, pornographic movie theaters, hourly hotels, and bars/discos with areas where sex is permitted - may be opportune sites for outreach; however, further research on SOPVs and the populations who frequent them is needed to inform such efforts. Methods. We conducted a cross-sectional online survey of adult MSM in Lima, Perú to evaluate patterns of SOPV attendance, associated sexual risk behaviors, and attitudes toward SOPV-based interventions. Participants were recruited through outreach to social media networks affiliated with local LGBTQ-aligned community groups. Our primary analytic objective was to estimate the association of HIVrelated sexual risk behaviors and SOPV attendance. Additionally, we performed exploratory analyses to describe risk behavior stratified by SOPV category and to examine the relationship between SOPV attendance and the use of online platforms to meet sex partners. Results. Overall, $389 \mathrm{MSM}$ completed the survey from November 2018 through May 2019, of whom 68\% reported attending an SOPV in the last three months. SOPV attendance was associated with multiple sexual risk behaviors, including transactional sex, group sex, substance use around the time of sex, and higher number of partners. Over two thirds of SOPV attendees indicated they would accept HIV testing if offered at SOPVs. Conclusions. SOPV attendance was common among MSM in Lima who participated in our survey, and SOPV attendees reported significantly greater engagement in sexual risk behaviors related to HIV transmission. Attitudes toward hypothetical SOPV-based interventions were generally favorable. These findings suggest that outreach at SOPVs may be an effective mechanism for reaching a particularly high-risk subpopulation of MSM in Perú to deliver targeted HIV testing and prevention interventions.

\section{Background}

As in most of the Americas, the HIV epidemic in Perú is concentrated among men who have sex with men (MSM) and transgender women (TW). Prevalence among these groups countrywide in 2018 was reported to be $12 \%$ and $16 \%$, respectively (1), although in the capital city of Lima it may be substantially higher, with estimates ranging from 18-20\% among MSM and 17-30\% among TW (2-6). In addition, high HIV incidence persists, with some studies reporting rates of $>10$ per 100 person-years $(3,4,7)$. Most notable, however, is the fact that HIV incidence in Perú has not significantly changed over the last decade despite the broad scale-up of antiretroviral treatment (ART) access during this time. While UNAIDS estimates suggest a stable overall incidence rate of 0.9 (confidence interval [Cl] $0.7-1.8$ ) per 100 personyears in 2010 compared to 1.1 (Cl 0.5 - 1.6) in 2017 (1), data from the Peruvian Ministry of Health show a $27 \%$ increase (from 4677 to 5926 cases) in new HIV diagnoses over this same time period (8). Since 2004 the Ministry of Health has provided antiretroviral treatment (ART) at no cost to individuals with HIV infection who meet clinical and immunologic criteria (9), and has moved to extend ART coverage to all 
Peruvians living with HIV following the release of the World Health Organization (WHO) "treat all" recommendations in 2015 (10-12). In contrast to this expansion in access to ART for HIV treatment, the use of antiretroviral drugs for pre-exposure prophylaxis (PrEP) is not yet subsidized in Perú and access remains quite limited, with fewer than 1,500 active PrEP users estimated in the country currently (13).

Delayed diagnosis of HIV infection is an important factor fueling onward sexual transmission in Perú (14-17). One recent study estimated that just $24 \%$ of MSM and TW living with HIV in Perú have been diagnosed, and among these only half are linked to care and on ART (18) - falling far short of the UNAIDS "90-90-90" targets (19). To close these gaps in Perú's HIV services continuum, new approaches are needed that more effectively engage vulnerable MSM and TW. Strategies that aim to address the underlying psychological and structural barriers experienced by members of these communities including stigma, fear, low risk perception, and geographic factors (20-23) - are most likely to have success.

Previous research suggests that offering HIV testing at community-based venues can reach individuals who may not otherwise access traditional clinic-based services (24-28), including in Perú $(5,7,29)$. Sexon-premise venues (SOPVs), which are social venues that provide a space to meet and have sex with other patrons, may be fitting sites for such outreach. Although sex is the primary, and often overtly stated, purpose of many SOPVs (e.g. sex clubs, pornographic movie theaters, and most saunas/bathhouses), the term also encompasses commercial establishments where sex is permitted or encouraged, even if not explicitly endorsed as its reason for being (e.g. hourly hotels and bars/discos with dark rooms) $(30,31)$. Studies from high-income countries (HIC) with concentrated HIV epidemics affecting MSM, including Australia and the United States, have demonstrated an association between SOPV attendance and highrisk sexual behavior (32-36). In addition, the feasibility of delivering sexual health interventions at SOPVs has been well documented in HIC settings, including programs offering safer sex counseling, free condom distribution, on-site testing for HIV and other sexually transmitted infections (STI), and distribution of HIV self-testing kits (37-47). While intervention uptake among SOPV patrons was relatively modest in two studies that assessed this outcome (uptakje was 24\% (37) and 51\% (39), respectively), several studies demonstrated SOPV-based HIV/STI testing to be effective at reaching individuals at high risk, including those who may not otherwise come to be tested in healthcare facilities. In contrast to the evidence base from HICs, research related to SOPVs and the populations who attend them in low- and middle-income countries (LMIC) such as Perú remains extremely limited.

Despite the paucity of evidence on the role of SOPVs in Perú's HIV epidemic - and in the Latin American context more broadly - several factors suggest that SOPVs may be ideal sites at which to conduct outreach to Peruvian MSM and TW at highest risk for HIV infection. MSM and TW in Perú commonly live at home with their family of origin $(48,49)$, where conservative social norms $(50)$ and stigma related to gender and sexuality $(51-53)$ can create powerful disincentives to bringing sex partners home. By functioning as alternative locations where MSM and TW can go to meet sex partners, SOPVs may play a fundamentally different - and relatively more important - role in Perú as compared to regions with more progressive social norms, such as those in which the existing literature is based. Additionally, the use of 
online platforms that facilitate efficient identification of sex partners - including geosocial networking apps - is increasing in Perú (49). SOPVs may be important meeting places for sex after identifying a prospective partner online. Importantly, SOPVs are by definition physical locations where individuals gather. Thus, in contrast to internet-based outreach, SOPVs are sites where HIV testing and biomedical prevention interventions could be delivered directly. Several recent studies among MSM and TW in Lima demonstrated high uptake of HIV testing, as well as high rates of new HIV diagnoses, at bars and other public social venues - including some SOPVs $(5,7,29)$. However, we were unable to identify any dedicated research from Perú - or any other Latin American country - focusing specifically on SOPVs or their clientele. One possible explanation is that SOPVs are only now being appreciated and their importance emphasized in the context of the surging popularity in the use of online platforms to meet sex partners. In order to better understand the role of SOPVs within high-risk sexual networks in Perú, and to inform future SOPV-based outreach strategies to deliver HIV testing and prevention interventions, we conducted an online survey of MSM and TW in Lima. The goals of this study were to evaluate the prevalence and patterns of SOPV attendance, the association of SOPV attendance with sexual risk behaviors, and the attitudes toward potential SOPV-based interventions in these communities.

\section{Methods}

\section{Study Population and Design}

We conducted a cross-sectional, internet-based survey among MSM and TW in Lima, Perú. Adults 18 years of age or greater who identified as either MSM or TW were eligible. We recruited participants by disseminating a link to the survey via social media platforms affiliated with a local LGBTQ-aligned community-based organization in Lima. An initial landing page included the consent form, basic instructions, and eligibility criteria. This was formatted for both desktop and mobile access, enabling survey participation from any computer or mobile device with an internet connection. Prior to accessing the survey, individuals were required to provide an electronic signature as an attestation of their eligibility and informed consent. Participation was completely anonymous and no incentive was offered. The study underwent bioethics review and received approvals from both the Vía Libre Comité Institucional de Bioética (Lima, Perú) and the University of Washington Institutional Review Board (Seattle, USA).

\section{Survey Instrument}

REDCap (54) was used for survey instrument development and administration, as well as data collection and storage. The survey included a total of 19 main questions, some of which branched to sub-questions based on the response (Additional File 1). Depending on the number of sub-questions prompted (based on answers to the main questions), survey completion took approximately 10-15 minutes. We collected basic demographic data and asked participants about venue attendance and sexual behaviors in the past 3 months, including how/where they met their recent partners and where they went to have sex, as well as online platforms used to meet partners. In addition to questions about SOPV attendance and sexual behaviors in general, we collected more detailed venue-specific data for participants who reported sex at 
an SOPV with either their last or penultimate partner (in the last 3 months). This included information about the physical and environmental features of the venue(s) they attended, sexual behaviors there (both observed and participated, including condom use), and attitudes toward hypothetical venue-based sexual health interventions such as condom/lubricant distribution and point-of-care testing for HIV and other sexually transmitted infections (STIs).

\section{Statistical Analysis}

After reaching our target of 400 completed surveys, data were exported from REDCap into Stata version 15 (StataCorp. 2017. Stata Statistical Software: Release 15. College Station, TX: StataCorp LLC) for analysis. We used descriptive statistics to summarize the proportion of participants who reported attending an SOPV in the last 3 months, as well as participant characteristics, including demographics, sexual behaviors (including the overall proportion who used an online platform to meet a sex partner in the last 3 months), knowledge of biomedical HIV prevention modalities (e.g. PrEP, “ $U=U$ ”), and attitudes toward venue-based HIV testing and prevention interventions, and other venue-specific factors. The objective of our primary analysis was to evaluate the relationship between sexual risk behaviors and SOPV attendance in the previous three months. We defined an SOPV as any of the following 5 venue categories: sauna, hotel, sex club, pornographic movie theater, or bar/disco known to permit sex. Additionally, we defined two categories of SOPV attendance: meeting a partner at, and having sex at, an SOPV. We estimated the crude prevalence ratio (PR) and 95\% confidence interval (Cl) for each factor of interest (all of which were coded as dichotomous variables), comparing the proportion with a given factor among participants who reported meeting a partner at (or having sex at) an SOPV in the previous three months to the proportion among those who did not. To further examine patterns of SOPV attendance and use of online platforms to identify sex partners, we conducted several exploratory sub-analyses, stratifying by SOPV category, using the detailed venue-level data collected on the last two partners. All statistical testing to estimate confidence intervals and prevalence ratios used the Chi-squared distribution. Statistical testing to assess for differences in proportions used either the Chi-squared or Fisher's exact test.

\section{Results}

From November 26, 2018 through May 16, 2019 a total of 389 MSM and 8 TW completed the online survey. Two transgender male and two cisgender female identifying individuals also completed a survey but were excluded from the analysis given they did not meet predetermined inclusion criteria. Additionally, because the low number of TW participants was insufficient to draw meaningful inferences for this important but distinct sub-population (55), we restricted our analysis to cisgender MSM only.

\section{Demographic and Behavioral Characteristics}

Among 389 MSM, 77\% identified as homosexual, $19 \%$ as bisexual, and $3 \%$ as heterosexual (Table 1 ). Median age was 30 years (interquartile range [IQR] 25 - 37), and just under half (47\%) had a university degree or higher (likely a reflection of the social media sites used for recruitment). Over three quarters 
lived with at least one family member, and more than half lived with one or both parents. Participants reported a median total number of sex partners of $3($ IQR $2-5)$ in the last 3 months. Nearly half reported sex under the influence of either drugs or alcohol, about one third participated in group sex, and about a quarter reported transactional sex (either received payment or paid for sex); however, only $2 \%$ identified as a sex worker. Overall, $85 \%$ reported ever being tested for HIV and $26 \%$ reported being HIV positive, the vast majority (94\%) of whom indicated they were taking ART. When given a statement illustrating the concept of " $\mathrm{U}=\mathrm{U}$ " (i.e. that sexual transmission of HIV is virtually impossible from an HIV-infected person with an undetectable viral load on ART), roughly half $(53 \%)$ indicated this was true. A somewhat larger proportion (73\%) reported that they had ever heard of PrEP.

Table 1. Demographic Characteristics, Sexual Behavior, and HIV Testing History $(\mathrm{N}=389)$

\begin{tabular}{|c|c|c|c|}
\hline \multicolumn{2}{|c|}{ Panel A. Demographic Characteristics } & \multicolumn{2}{|c|}{ Panel B. Sexual Behaviors } \\
\hline Aqe (years) & $\frac{\text { Median }}{30(25-37)}$ & $\geq 1$ sex partner & $\frac{n}{365(94 \%)}$ \\
\hline Monthly income (Soles) ${ }^{\mathrm{a}}$ & $\begin{array}{c}1500(700- \\
3000)\end{array}$ & Had "casual" partner & $215(55 \%)$ \\
\hline & & Group sex & 141 (36\%) \\
\hline & $\underline{\mathrm{n}}(\%$ of $\mathrm{N})$ & Transactional sex & $110(28 \%)$ \\
\hline Highest Education & & Paid for sex & $67(17 \%)$ \\
\hline Secondary school or less & 152 (39\%) & Was paid for sex & 57 (15\%) \\
\hline Technical/Vocational & 56 (14\%) & Identifies as sex worker & $8(2 \%)$ \\
\hline University degree or more & $181(47 \%)$ & $\begin{array}{l}\text { Substance use associated } \\
\mathrm{w} / \text { sex }\end{array}$ & $172(44 \%)$ \\
\hline Living Situation $\mathrm{b}$ & - & Alcohol & $144(37 \%)$ \\
\hline Lives alone & $62(16 \%)$ & Marijuana & 66 (17\%) \\
\hline With a friend / roommate & $34(9 \%)$ & Poppers & $47(12 \%)$ \\
\hline With spouse / stable partner & $56(14 \%)$ & Other & $10(3 \%)$ \\
\hline With any family member ${ }^{\mathrm{C}}$ & $301(77 \%)$ & Condomless anal sex ${ }^{\mathrm{d}}$ & $176(45 \%)$ \\
\hline Sexual Identity/Orientation & & Receptive & $121(31 \%)$ \\
\hline Homosexual & 299 (77\%) & Insertive & $118(30 \%)$ \\
\hline Bisexual & $74(19 \%)$ & HIV Prevention Knowledge & \\
\hline Heterosexual & $13(3 \%)$ & Ever heard of PrEP & 285 (73\%) \\
\hline Pansexual & $3(<1 \%)$ & Believes that " $U=U$ " is true ${ }^{f}$ & $208(53 \%)$ \\
\hline \multicolumn{4}{|c|}{ Panel C. HIV Testing History } \\
\hline HIV-positive & $\frac{\mathrm{n}}{102(\% \text { of } N)}$ & $\overline{\text { Last negative HIV test }}$ & $\underline{\mathrm{n}}(\%$ of $\mathrm{N}=227 \mathrm{HIV}-)$ \\
\hline On ART, n (\% of N=102 HIV+) & $96(94 \%)$ & $<3$ months ago & 89 (39\%) \\
\hline $\begin{array}{l}\text { Unknown HIV status / Never } \\
\text { tested }\end{array}$ & $49(13 \%)$ & 3-6 months ago & $54(24 \%)$ \\
\hline Declined to disclose HIV status & $11(3 \%)$ & 6-12 months ago & $42(19 \%)$ \\
\hline HIV-negative when last tested & 227 (58\%) & $\geq 12$ months ago & $41(18 \%)$ \\
\hline
\end{tabular}

MSM: men who have sex with men; IQR: interquartile range; ART: antiretroviral treatment; PrEP: pre-exposure prophylaxis; ${ }^{\mathrm{a} E x c h a n g e ~ r a t e ~ o n ~} 26$ Nov 2018: 1 USD = 3.37 Peruvian Nuevo Soles (56); ${ }^{b}$ Categories not mutually exclusive; C Specific family members: Mother 190 (49\%); Father 129 (33\%); Sibling 166 (43\%); Aunt/Uncle 50 (13\%); Cousin 34 (9\%); Grandparent 31 (8\%); Niece/Nephew 5 (1\%); Son/Daughter 3 (<1\%); Brother/Sister-in-Law 3 (<1\%); ${ }^{\mathrm{d}}$ Refers to last or penultimate partner; ${ }^{\mathrm{e}}$ Other drugs: Cocaine 5 (1\%); Ecstasy 4 (1\%); Amphetamine $2(<1 \%)$; Heroin 1

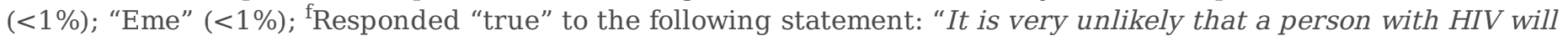
transmit the virus to their sexual partner if the person with HIV is taking antiretroviral therapy and the virus is undetectable in their blood" 
Recent SOPV attendance was common, as was the use of online platforms to meet a sex partner (Table 2). Overall, $68 \%$ reported either meeting a partner or having sex at an SOPV at least once in the last 3 months (42\% met a partner at an SOPV and $61 \%$ had sex at an SOPV). The most common SOPV category for meeting a new partner was bars/discos, while the most frequently cited SOPV category for having sex was hotels. The use of online platforms to meet partners was also common: overall, $78 \%$ reported meeting a partner online in the last 3 months (median 3 online partners, IQR $1-5$ ). Grindr was by far the most frequently reported online platform, followed by Facebook and WhatsApp.

Table 2. SOPV Attendance and Online Platform Use ( $\mathrm{N}=389)$

SOPVs Attended to Meet a Partner or Have Sex in Last 3 Months

$\begin{array}{lccc} & \text { MET Partner or } & \underline{n}(\% \text { of N) } & \text { Had SEX } \\ \text { SEX at SOPV } & \text { MET Partner at SOPV } & 236(61 \%) \\ \text { Any SOPV } & 264(68 \%) & 165(42 \%) & 193(50 \%) \\ \text { Hotel } & 193(50 \%) & 3(<1 \%) & 34(9 \%) \\ \text { Bar/Disco } & 109(28 \%) & 95(24 \%) & 84(22 \%) \\ \text { Sauna } & 98(25 \%) & 90(23 \%) & 29(7 \%) \\ \text { Sex Club } & 40(10 \%) & 36(9 \%) & 15(4 \%) \\ \text { Porno Theater } & 18(5 \%) & 15(4 \%) & \end{array}$

Online Platforms Used to Meet a Sex Partner in Last 3 Months

\begin{tabular}{lc}
\hline & $\mathrm{n}(\%$ of N) \\
Met $\geq 1$ partner online & $304(78 \%)$ \\
Grindr & $217(56 \%)$ \\
Facebook & $84(22 \%)$ \\
WhatsApp group & $42(11 \%)$ \\
Manhunt & $28(7 \%)$ \\
Other & $72(19 \%)$ \\
\hline
\end{tabular}

SOPV: sex-on-premise venue; a Other online platforms, n (\%): Tinder 20 (5\%); Scruff 18 (5\%); "Chat" (e.g.

PeruGayChat, GayChat, ChatGay, ChatPeruGay, ElChat) 10 (3\%); Instagram 8 (2\%); GayRomeo 1 (<1\%); Surge 1 $(<1 \%)$

Association of SOPV Attendance and Sexual Risk Characteristics

SOPV attendees, as compared to MSM who did not attend an SOPV in the last 3 months, were more likely to report several sexual risk behaviors, including group sex, transactional sex, sex under the influence of alcohol, sex with a "casual" partner, and $\geq 3$ sex partners in the last 3 months; these associations were statistically significant in all cases regardless of whether evaluated with respect to meeting a partner or to having sex at an SOPV (Table 3). SOPV attendees were somewhat more likely to report having ever been tested for HIV. However, self-reported HIV seropositivity was not associated with SOPV attendance. Meeting a partner at an SOPV, but not having sex at an SOPV, was associated with older age and higher monthly income. Having sex at an SOPV, but not meeting a partner at an SOPV, was associated with living with family and with having a recent online partner. We performed sensitivity analyses excluding 
self-reported HIV-positive individuals, which did not substantively alter our findings regarding the relationship between SOPV attendance and sexual risk behaviors.

Table 3. Factors Associated with SOPV Attendance in the Last 3 Months

\begin{tabular}{|c|c|c|c|}
\hline \multirow[b]{2}{*}{ Demographic Characteristics } & \multirow{2}{*}{$\begin{array}{c}\text { \% of } \mathrm{N} \\
\text { Overall } \\
\text { Prevalence } \\
(\mathrm{N}=389)\end{array}$} & \multicolumn{2}{|c|}{ Prevalence Ratio (95\% CI) } \\
\hline & & $\begin{array}{c}\text { MET a partner } \\
\geq 1 \mathrm{X} \text { at SOPV } \\
(\mathrm{n}=165)\end{array}$ & $\begin{array}{c}\text { Had SEX } \\
\geq 1 \mathrm{X} \text { at SOPV } \\
(\mathrm{n}=236)\end{array}$ \\
\hline Age $\geq 30$ years & $51 \%$ & $1.28(1.05-1.55)$ & $1.11(0.90-1.36)$ \\
\hline Bisexual identifying & $19 \%$ & $0.93(0.61-1.41)$ & $0.90(0.59-1.36)$ \\
\hline Monthly income $\geq 1500$ Soles $^{a}$ & $52 \%$ & $1.21(1.00-1.46)$ & $1.12(0.91-1.36)$ \\
\hline Lives with any family member & $77 \%$ & $1.03(0.93-1.15)$ & $1.16(1.03-1.30)$ \\
\hline University education & $47 \%$ & $1.08(0.87-1.33)$ & $1.05(0.84-1.31)$ \\
\hline \multicolumn{4}{|l|}{ Sexual Behaviors in Last 3 Months } \\
\hline$\geq 3$ sex partners & $54 \%$ & $2.00(1.65-2.41)$ & $1.92(1.52-2.43)$ \\
\hline Met $\geq 1$ partner online & $78 \%$ & $0.96(0.86-1.07)$ & $1.13(1.01-1.26)$ \\
\hline Group sex & $36 \%$ & $2.12(1.62-2.79)$ & $1.70(1.25-2.31)$ \\
\hline Transactional sex & $28 \%$ & $1.63(1.19-2.24)$ & $2.45(1.62-3.70)$ \\
\hline Had "casual" partnerb & $55 \%$ & $1.42(1.19-1.69)$ & $1.50(1.21-1.84)$ \\
\hline Condomless anal sex ${ }^{b}$ & $45 \%$ & $1.18(0.95-1.47)$ & $1.19(0.94-1.50)$ \\
\hline Substance use associated $\mathrm{w} / \mathrm{sex}$ & $44 \%$ & $1.64(1.31-2.04)$ & $1.67(1.29-2.18)$ \\
\hline Alcohol & $37 \%$ & $1.65(1.27-2.14)$ & $1.69(1.25-2.28)$ \\
\hline Marijuana & $17 \%$ & $1.73(1.11-2.70)$ & $1.21(0.76-1.93)$ \\
\hline Poppers & $12 \%$ & $2.90(1.62-5.17)$ & $2.74(1.36-5.50)$ \\
\hline \multicolumn{4}{|l|}{ HIV Testing History } \\
\hline Ever had an HIV test & $85 \%$ & $1.09(1.01-1.18)$ & $1.11(1.02-1.22)$ \\
\hline HIV positive & $26 \%$ & $1.36(0.97-1.89)$ & $0.96(0.69-1.35)$ \\
\hline
\end{tabular}

Statistically significant associations $(\mathrm{p}<0.05)$ denoted in bold; SOPV: sex-on-premise venue; ${ }^{\mathrm{a}}$ Exchange rate on 26 Nov 2018: 1 USD = 3.37 Peruvian Nuevo Soles (56); ${ }^{\text {b }}$ Refers to last or penultimate partner

\section{Sexual Risk Behaviors and Online Platform Use Stratified by SOPV Category}

Stratification by SOPV category revealed heterogeneity in the sexual risk behaviors reported by populations of MSM as defined by attendance of different categories of venue to either meet a partner or have sex (Table 4). For this exploratory sub-analysis, we define SOPV attendance as either meeting a partner or having sex at a given type of SOPV and we report only descriptive statistics, as participants could indicate they attended multiple venues, precluding the use of inferential statistical tests. 


\begin{tabular}{|c|c|c|c|c|c|c|}
\hline \multirow[b]{2}{*}{ Risk Characteristic ${ }^{a}$} & \multicolumn{6}{|c|}{$\underline{\mathrm{n}}(\underline{\% \text { of } N})$} \\
\hline & $\begin{array}{c}\text { Any SOPV } \\
(\mathrm{N}=264)\end{array}$ & $\begin{array}{c}\text { Hotel } \\
(\mathrm{N}=193)\end{array}$ & $\begin{array}{c}\text { Sauna } \\
(\mathrm{N}=98)\end{array}$ & $\begin{array}{l}\text { Sex Club } \\
(\mathrm{N}=40)\end{array}$ & $\begin{array}{l}\text { Porno Theater } \\
(\mathrm{N}=18)\end{array}$ & $\begin{array}{c}\text { Bar/Disco } \\
(\mathrm{N}=109)\end{array}$ \\
\hline$\geq 3$ sex partners & $172(65 \%)$ & $124(65 \%)$ & $83(85 \%)$ & $37(93 \%)$ & $18(100 \%)$ & $75(69 \%)$ \\
\hline Group sex & $111(42 \%)$ & $74(38 \%)$ & $59(60 \%)$ & $28(70 \%)$ & $13(72 \%)$ & $54(50 \%)$ \\
\hline Transactional sex & 91 (34\%) & $70(36 \%)$ & $34(35 \%)$ & $21(53 \%)$ & $12(67 \%)$ & $38(35 \%)$ \\
\hline “Casual” partnerb & $165(63 \%)$ & $119(62 \%)$ & $69(70 \%)$ & $30(75 \%)$ & $13(72 \%)$ & $70(64 \%)$ \\
\hline Condomless anal sex ${ }^{b}$ & $126(48 \%)$ & $93(48 \%)$ & $52(53 \%)$ & $15(38 \%)$ & $10(56 \%)$ & $54(50 \%)$ \\
\hline Substance use w/sex & $142(54 \%)$ & $102(53 \%)$ & $52(53 \%)$ & $24(60 \%)$ & 8 (44\%) & $68(62 \%)$ \\
\hline HIV positive & $69(26 \%)$ & $42(22 \%)$ & $33(34 \%)$ & $16(40 \%)$ & $7(39 \%)$ & $33(30 \%)$ \\
\hline
\end{tabular}

SOPV attendance is defined as either meeting a partner or having sex in the last 3 months; categories (columns) not mutually exclusive. SOPV: sex-on-premise venue; ${ }^{a}$ Refers to within the last 3 months (where applicable); ${ }^{b}$ Refers to last or penultimate partner

Among participants who met their last or penultimate partner online, we tabulated the SOPV categories they attended to have sex with that same partner. Of 241 participants who met their last partner online, 66 $(27 \%)$ had sex at a hotel, $4(2 \%)$ at a sauna, and $2(<1 \%)$ at a bar or disco; none had sex at a porno theater or sex club. Of 173 participants who met their penultimate partner online, $54(31 \%)$ had sex at a hotel, 2 $(1 \%)$ at a sauna, $1(<1 \%)$ at a sex club, and $1(<1 \%)$ at a bar/disco; none had sex at a porno theater. Overall, compared to those who did not meet a partner online, participants who met any recent partner online were more likely to report sex at a hotel (54\% among those with a recent online partner vs 34\% among those with no recent online partner, $p=0.001)$ or at a bar/disco $(11 \% \mathrm{vs} 2 \%, \mathrm{p}=0.016)$ in the last 3 months, but not at a sauna ( $22 \%$ vs $21 \%, p=0.916)$, sex club ( $8 \%$ vs $6 \%, p=0.532)$, or porno theater $(4 \%$ vs $5 \%, p=0.749)$.

\section{Sexual Risk at SOPVs and Attitudes Toward SOPV-Based Interventions}

For MSM who had sex at an SOPV with at least one recent partner $(\mathrm{N}=177)$, we tabulated venue-specific data on sexual encounters with their last $(n=138)$ and/or penultimate $(n=113)$ partners. Participants indicated that condoms were available at SOPVs for $49 \%$ of sexual encounters overall, including $48 \%$ at hotels, $57 \%$ at saunas, $76 \%$ at sex clubs, $25 \%$ at porno theaters, and $0 \%$ at bars/discos. Lube was available at $27 \%$ of the encounters $(29 \%$ at hotels, $29 \%$ at saunas, $13 \%$ at sex clubs, $0 \%$ at porno theaters, and $50 \%$ at bars/discos). Alcohol was present for $29 \%$ of sexual encounters at SOPVs overall $(24 \%$ at hotels, $43 \%$ at saunas, $75 \%$ at sex clubs, $0 \%$ at porno theaters, and $50 \%$ at bars/discos) and group sex occurred at $20 \%$ of encounters ( $12 \%$ at hotels, $33 \%$ at saunas, $75 \%$ at sex clubs, $50 \%$ at porno theaters, and $50 \%$ at bars/discos). Attitudes toward hypothetical SOPV-based sexual health interventions such as $\mathrm{HIV/STI}$ testing and condom/lube distribution varied based on the category of SOPV in question, but generally indicated a high level of acceptability (Figure 1).

\section{Discussion}


More than two thirds of our study participants attended an SOPV either to meet a sex partner or to have sex in the last 3 months, suggesting that SOPV attendance may be quite common among MSM in Lima. In addition, those who attended an SOPV were significantly more likely to report behaviors associated with elevated risk of HIV and STI transmission, including group sex, transactional sex, sex under the influence of alcohol, sex with a casual partner, and more overall partners. Notably, the majority of SOPV attendees indicated favorable attitudes toward theoretical SOPV-based sexual health interventions such as condom/lubricant distribution and HIV/STI testing.

Although SOPV attendance was associated with several sexual risk behaviors, one notable exception was condomless anal sex. One possible explanation is that we asked questions about sexual positioning and condom use with reference to only the last two partners, rather than asking about al/ recent partners (as was asked for other behaviors). However, similar results to ours were observed in a large online survey conducted in the mid-2000s of MSM in the US, which found that meeting a partner at a physical venue (e.g. bars/clubs, bathhouses, and public outdoor spaces) was associated with risk behaviors such as alcohol use - but not with condomless anal sex (33). In our exploratory analyses, sexual risk behavior also appeared to vary by SOPV category. For example, compared with other categories, the proportion of MSM reporting nearly every risk characteristic (except condomless anal sex) was higher for sex clubs (Table 4). In contrast, hourly hotels, which were by far the most common SOPV attended, appeared to be frequented by lower risk clientele. Taken together, these results suggest that more nuanced data collection may be necessary to characterize sexual risk behaviors associated with SOPV attendance in this setting. Such information, including the identification of specific SOPVs attended by the highest risk clientele, would be particularly germane to the development of SOPV-based outreach strategies to deliver HIV testing and other sexual health interventions.

The vast majority $(78 \%)$ of our sample population reported meeting a sex partner online in the last 3 months. This is consistent with observations from a large contemporaneous online survey of MSM in Brazil, Mexico, and Perú conducted in 2018 by Torres et al, which found that $81 \%$ of Peruvian MSM reported using apps for sexual encounters (57). Notably, these estimates are roughly double what was found in two studies of MSM in Lima from just a few years ago, including one conducted in 2013-2014 (in person) and another in 2012-2013 (online), which reported $37 \%$ and $44 \%$ of participants had a recent online partner, respectively $(3,49)$. Together these findings likely reflect the rapid expansion in recent years of access to low-cost internet services and mobile devices in Perú; however, the use of an online survey, which selects for individuals with internet access, may have also played a small role in the higher estimates observed in both our study and by Torres et al (57). In addition, geosocial networking applications (e.g. Grindr), which have been associated with increased sexual risk behavior and STIs incidence in some settings (58-60), have had substantial growth in popularity since the two earlier studies.

Our results also shed light on the relationship between online platform use and SOPV attendance. MSM who had sex at an SOPV, but not those who met a partner at an SOPV, were significantly more likely to report having a recent online partner. This mirrored what we observed for one's living situation: living with

Page $10 / 20$ 
family was associated having sex at an SOPV but not meeting a partner at one. One potential explanation for this pattern is that, although online platforms can facilitate the identification of sex partners, stigma precludes those who live with family from having sex with these partners at home. SOPVs might fill this void by offering places outside of the home where MSM can have sex with online partners. We also found that, when stratified by SOPV category, the association between SOPV attendance and online platform use was significant only for hotels and bars/discos, suggesting that these venues may be preferred rendezvous sites for meeting online partners for sex, perhaps because identifying new sex partners in person may be easier at saunas, sex clubs, and porno theaters.

The primary limitation of this study relates to its generalizability. Our sampling method, which relied on recruitment through local social media networks, selected a population that is somewhat more educated and affluent than the general population of MSM in Lima. Therefore, our estimates of SOPV attendance and sexual behavior may not reflect the experiences of MSM in lower socioeconomic strata. Self-reported HIV prevalence was $26 \%$, which is within the range reported by other studies of MSM in Lima $(5,6)$. However, over $80 \%$ of MSM in this study reported having ever received an HIV test and over $90 \%$ of those who self-reported being HIV positive indicated they were taking ART. In both cases, these rates are somewhat higher than past population estimates of HIV testing and ART coverage, respectively, in the region $(1,18)$, suggesting above average access to health services. If so, the estimates derived from our sample population may in fact under-represent the risk profile of MSM in Lima on average. Future studies of SOPV attendance and associated sexual risk behaviors in Lima should consider utilizing alternative sampling strategies to include MSM in less affluent communities, as well as TW - an extremely vulnerable population that our online recruitment strategy did not adequately reach.

Although, overall, participants reported favorable attitudes toward SOPV-based HIV/STI testing interventions (Figure 1), interpretation of these data is limited by the small sample size for sex clubs, porno theaters, and bars/discos. The use of a survey to evaluate acceptability of hypothetical interventions may also overestimate uptake in real-world settings. Nevertheless, our results, which indicate that over two thirds of MSM would either "definitely" (45\%) or "probably" (23\%) accept SOPVbased HIV testing, are consistent with direct observations from the field, including a recent study that demonstrated $52 \%$ uptake of venue-based HIV testing when it was offered to MSM and TW at bars, clubs, and public parks in Lima (5).

Despite the limitations to its generalizability, the online administration of our survey allowed it to be conducted in a completely anonymous fashion. This significantly reduced the likelihood of social desirability bias and is an important strength of the study. While anonymous participation can also, in theory, enable a single person to complete more than one survey, there was no incentive to do so and completion of the survey was relatively time-intensive ( 10-15 minutes on average). Therefore, duplicate survey responses or other spurious results related to external incentives are unlikely.

In summary, our results support the suggestion that SOPVs play an important role in MSM sexual networks in Perú. This may be particularly pronounced compared to settings where young adults tend to 
leave the family home at a younger age, or where stigma is a less prominent driver of sexual decisionmaking. Both SOPV attendance and the use of online platforms to meet sex partners appear to be highly prevalent behaviors among MSM in Lima. Furthermore, our findings indicate that MSM who attend SOPVs may be at particularly high risk for HIV/STI transmission, underscoring an opportunity to work with SOPVs to develop interventions that will expand access to testing, treatment, and prevention services. Although access to PrEP is relatively limited at present time in Perú, as it expands partnerships with SOPVs could be leveraged to promote linkage to existing providers or even facilitate future medication distribution. Importantly, our results suggest SOPV-based interventions would be accepted by most MSM in Lima who frequent these venues. Overall, these findings have important public health implications and suggest that SOPVs in Lima are suitable sites for targeted HIV testing and prevention interventions.

\section{Conclusions}

Among MSM in Lima, SOPV attendance appears to be common and SOPV attendees may be at higher risk for HIV transmission compared to MSM in general. SOPV-based outreach may be an acceptable strategy to deliver sexual health interventions in this community, and SOPVs may be ideal points of contact where MSM in high-risk sexual networks - including those who meet partners online - could be reached for HIV testing. Our findings support further development of targeted SOPV-based interventions as a way to increase access to HIV testing, treatment, and prevention services for MSM in Perú.

\section{List Of Abbreviations}

MSM: who have sex with men

SOPV: sex-on-premise venue

TW: transgender women

WHO: World Health Organization

ART: antiretroviral treatment

PrEP: pre-exposure prophylaxis

STI: sexually transmitted infection

HIC: high-income country

LMIC: low/middle-income country

PR: prevalence ratio

Cl: confidence interval 
IQR: interquartile range

$\mathrm{U}=\mathrm{U}$ : undetectable equals untransmissible

\section{Declarations}

\section{Ethics approval and consent to participate}

This research underwent full review and was approved by the Vía Libre Comité Institucional de Bioética (3876 [2018a]) and the University of Washington Institutional Review Board (STUDY00005823). All study participants provided informed consent and attested to their eligibility in the form of an electronic signature prior to their participation.

\section{Consent for publication}

Not applicable.

\section{Availability of data and materials}

The datasets used and analyzed during the current study are available from the corresponding author on reasonable request.

\section{Competing interests}

The authors declare they have no competing interests.

\section{Funding}

This research was supported by a 2018 CFAR International Pilot Award from the University of Washington / Fred Hutch Center for AIDS Research (NIH AI027757); in addition, AL received support as a post-doctoral fellow under the University of Washington STD/AIDS Research Training Grant (NIH T32 Al07140). We also acknowledge funding support related to the use of REDCap to carry out this study (UL1 TR002319, KL2 TR002317, and TL1 TR002318 from NCATS/NIH). None of the funding bodies supporting this research had any role in the study design, data collection, analysis, or interpretation of results.

\section{Authors' contributions}

$A L, H S, J H, R C$, and $A D$ all made substantial contributions to the conception and study design for this work. In addition, AL obtained funding, drafted all study materials (including the survey instrument, consent form, and documents for bioethics review), developed and maintained the online survey in REDCap, performed all data analysis, interpreted the results, and drafted the manuscript; HS contributed substantially to development of the survey instrument and documents for bioethics review, led recruitment efforts, and provided substantive interpretation of the results; JH contributed substantially to the acquisition of data and interpretation of results; RC contributed substantially to the acquisition of 
data and interpretation of results; and AD contributed substantially to the development of study materials and interpretation of data, and provided substantive revision of the manuscript. All authors read and approved the final manuscript submitted for publication.

\section{Acknowledgements}

We wish to acknowledge the study participants who shared their experiences, time, and effort in order to make this work possible. In addition, we are grateful for the technical support of the REDCap administrative teams at the University of Washington Institute of Translational Health Sciences and the Fred Hutchinson Cancer Research Center.

\section{References}

1. Sidibe M. UNAIDS Data 2018. Program HIV/AIDS. 2018;1-376.

2. Silva-Santisteban A, Raymond HF, Salazar X, Villayzan J, Leon S, McFarland W, et al. Understanding the HIV/AIDS Epidemic in Transgender Women of Lima, Peru: Results from a Sero-Epidemiologic Study Using Respondent Driven Sampling. AIDS Behav. 2012 May 8;16(4):872-81.

3. Passaro RC, Haley CA, Sanchez H, Vermund SH, Kipp AM. High HIV prevalence and the internet as a source of HIV-related service information at a community-based organization in Peru: a crosssectional study of men who have sex with men. BMC Public Health. 2016 Dec 24;16(1):871.

4. Lama JR, Brezak A, Dobbins JG, Sanchez H, Cabello R, Rios J, et al. Design strategy of the sabes study: Diagnosis and treatment of early HIV infection among men who have sex with men and transgender women in Lima, Peru, 2013-2017. Am J Epidemiol. 2018;187(8):1577-85.

5. Allan-Blitz L-T, Herrera MC, Calvo GM, Vargas SK, Caceres CF, Klausner JD, et al. Venue-Based HIVTesting: An Effective Screening Strategy for High-Risk Populations in Lima, Peru. AIDS Behav. 2019 Apr 30;23(4):813-9.

6. Castillo R, Konda KA, Leon SR, Silva-Santisteban A, Salazar X, Klausner JD, et al. HIV and Sexually Transmitted Infection Incidence and Associated Risk Factors Among High-Risk MSM and Male-toFemale Transgender Women in Lima, Peru. JAIDS J Acquir Immune Defic Syndr. 2015 Aug;69(5):567-75.

7. Villaran M, Brezak A, Ahmed S, Ulrich A, Duerr A, Herbeck J, et al. A study of potential HIV transmission hotspots among men who have sex with men and transgender women in Lima, Peru. J Int AIDS Soc. 2016;19(Supplement 5):98-9.

8. Ministerio de Salud del Peru. Situación epidemiológica del VIH-Sida en el Perú. Bol VIH Mens del Cent Nac Epidemiol Prevención y Control Enfermedades. 2018;diciembre.

9. Vargas V. The new HIV/AIDS program in Peru: the role of prioritizing and budgeting for results. Unaids. 2015.

10. World Health Organization. Guideline on When To Start Antiretroviral Therapy and on Pre-Exposure Prophylaxis for HIV. 2015. 
11. Brazier E, Maruri F, Duda SN, Tymejczyk O, Wester CW, Somi G, et al. Implementation of "Treat-all" at adult HIV care and treatment sites in the Global leDEA Consortium: results from the Site Assessment Survey. J Int AIDS Soc. 2019 Jul 1;22(7):e25331.

12. World Health Organzation. Treat All: Policy Adoption and Implementation Status in Countries HIV Treatment and Care. HIV Treatment and Care Fact Sheet. 2017.

13. AIDS Vaccine Advocacy Coalition (AVAC). PrEPWatch [Internet]. [cited 2020 Feb 12]. Available from: https://www.prepwatch.org/country/peru/

14. Skarbinski J, Rosenberg E, Paz-Bailey G, Hall HI, Rose CE, Viall AH, et al. Human immunodeficiency virus transmission at each step of the care continuum in the United States. JAMA Intern Med. 2015;175(4):588-96.

15. Frieden TR, Foti KE, Mermin J. Applying Public Health Principles to the HIV Epidemic - How Are We Doing? Malina D, editor. N Engl J Med. 2015 Dec 3;373(23):2281-7.

16. Dimitrov D, Wood D, Ulrich A, Swan DA, Adamson B, Lama JR, et al. Projected effectiveness of HIV detection during early infection and rapid ART initiation among MSM and transgender women in Peru: A modeling study. Infect Dis Model. 2019;4:73-82.

17. Goodreau SM, Carnegie NB, Vittinghoff E, Lama JR, Sanchez J. What Drives the US and Peruvian HIV Epidemics in Men Who Have Sex with Men (MSM)? PLoS One. 2012;7(11):50522.

18. Chow JY, Konda KA, Borquez A, Caballero P, Silva-Santisteban A, Klausner JD, et al. Peru's HIV care continuum among men who have sex with men and transgender women: opportunities to optimize treatment and prevention. Int J STD AIDS. 2016 Oct 10;27(12):1039-48.

19. UNAIDS. Ending Aids Progress Towards the 90-90-90 Targets. Glob Aids Updat. 2017;198.

20. Lee SW, Deiss RG, Segura ER, Clark JL, Lake JE, Konda KA, et al. A cross-sectional study of low HIV testing frequency and high-risk behaviour among men who have sex with men and transgender women in Lima, Peru. BMC Public Health. 2015 Dec 21;15(1):408.

21. Blas MM, Alva IE, Cabello R, Carcamo C, Kurth AE. Risk Behaviors and Reasons for not Getting Tested for HIV among Men Who Have Sex with Men: An Online Survey in Peru. Thorne C, editor. PLoS One. 2011 Nov 9;6(11):e27334.

22. Rich KM, Huamaní JV, Kiani SN, Cabello R, Elish P, Arce JF, et al. Correlates of viral suppression among HIV-infected men who have sex with men and transgender women in Lima, Peru. AIDS Care Psychol Socio-Medical Asp AIDS/HIV. 2018 May 23;30(11):1341-50.

23. Krueger EA, Chiu CCJ, Menacho LA, Young SD. HIV testing among social media-using Peruvian men who have sex with men: correlates and social context. AIDS Care - Psychol Socio-Medical Asp AIDS/HIV. 2016 Oct 2;28(10):1301-5.

24. Sharma M, Ying R, Tarr G, Barnabas R. Systematic review and meta-analysis of community and facility-based HIV testing to address linkage to care gaps in sub-Saharan Africa. Nature. 2015;528(7580):S77-85.

25. Raj A, Yore J, Urada L, Triplett DP, Vaida F, Smith LR. Multi-Site Evaluation of Community-Based Efforts to Improve Engagement in HIV Care Among Populations Disproportionately Affected by HIV in 
the United States. AIDS Patient Care STDS. 2018;32(11):438-49.

26. Suthar AB, Ford N, Bachanas PJ, Wong VJ, Rajan JS, Saltzman AK, et al. Towards Universal Voluntary HIV Testing and Counselling: A Systematic Review and Meta-Analysis of CommunityBased Approaches. Sansom SL, editor. PLoS Med. 2013 Aug 13;10(8):e1001496.

27. Smith L V., Rudy ET, Javanbakht M, Uniyal A, Sy LS, Horton T, et al. Client Satisfaction with Rapid HIV Testing: Comparison Between an Urban Sexually Transmitted Disease Clinic and a CommunityBased Testing Center. AIDS Patient Care STDS. 2006 Oct 19;20(10):693-700.

28. Campbell CK, Lippman SA, Moss N, Lightfoot M. Strategies to Increase HIV Testing Among MSM: A Synthesis of the Literature. AIDS Behav. 2018 Aug 17;22(8):2387-412.

29. Lipsitz MC, Segura ER, Castro JL, Smith E, Medrano C, Clark JL, et al. Bringing testing to the people benefits of mobile unit HIV/syphilis testing in Lima, Peru, 2007-2009. Int J STD AIDS. 2014 Apr $9 ; 25(5): 325-31$.

30. AIDS Council of New South Wales. Sex on premises venue code of practice. 2015.

31. Frank K. Rethinking Risk, Culture, and Intervention in Collective Sex Environments. Arch Sex Behav. 2019;48(1):3-30.

32. Lyons A, Smith AMA, Grierson JW, Von Doussa H. Australian mens sexual practices in saunas, sex clubs and other male sex on premises venues. Sex Health. 2010;7(2):186-92.

33. Grov C, Hirshfield S, Remien RH, Humberstone M, Chiasson MA. Exploring the venue's role in risky sexual behavior among gay and bisexual men: An event-level analysis from a national online survey in the U.S. Vol. 42, Archives of Sexual Behavior. Springer US; 2013. p. 291-302.

34. Grov C. HIV Risk and substance use in men who have Sex with men surveyed in bathhouses, bars/clubs, and on Craigslist.org: Venue of recruitment matters. AIDS Behav. 2012;16(4):807-17.

35. Meunier É, Siegel K. Sex club/party attendance and STI among men who have sex with men: Results from an online survey in New York City. Sex Transm Infect. 2019;(November 2016):1-4.

36. Binson D, Woods WJ, Pollack L, Paul J, Stall R, Catania JA. Differential HIV Risk in Bathhouses and Public Cruising Areas. Am J Public Health. 2001;91(9):1482-6.

37. Lister NA, Smith A, Tabrizi S, Hayes P, Medland NA, Garland S, et al. Screening for Neisseria gonorrhoeae and Chlamydia trachomatis in Men Who Have Sex with Men at Male-only Saunas. Sex Transm Dis. 2003;30(12):886-9.

38. Spielberg F, Branson BM, Goldbaum GM, Kurth A, Wood RW. Designing an HIV Counseling and Testing Program for Bathhouses. J Homosex. 2003;44(3-4):203-20.

39. Woods WJ, Lippman SA, Agnew E, Carroll S, Binson D. Bathhouse distribution of HIV self-testing kits reaches diverse, high-risk population. AIDS Care - Psychol Socio-Medical Asp AIDS/HIV. 2016;28:111-3.

40. Debattista J, Dwyer J, Anderson R, Rowling D, Patten J, Mortlock M. Screening for syphilis among men who have sex with men in various clinical settings. Sex Transm Infect. 2004 Dec 1;80(6):505-8. 
41. Ko N-Y, Lee H-C, Hung C-C, Chang J-L, Lee N-Y, Chang C-M, et al. Effects of structural intervention on increasing condom availability and reducing risky sexual behaviours in gay bathhouse attendees. AIDS Care. 2009 Dec 12;21(12):1499-507.

42. Huebner DM, Binson D, Woods WJ, Dilworth SE, Neilands TB, Grinstead O. Bathhouse-Based Voluntary Counseling and Testing Is Feasible and Shows Preliminary Evidence of Effectiveness. JAIDS J Acquir Immune Defic Syndr. 2006 Oct;43(2):239-46.

43. Daskalakis D, Silvera R, Bernstein K, Stein D, Hagerty R, Hutt R, et al. Implementation of HIV Testing at 2 New York City Bathhouses: From Pilot to Clinical Service. Clin Infect Dis. 2009;48(11):1609-16.

44. Birrell F, Staunton S, Debattista J, Roudenko N, Rutkin W, Davis C. Pilot of non-invasive (oralfluid) testing for HIV within a community setting. Sex Health. 2010;7:11-6.

45. Woods WJ, Euren J, Pollack LM, Binson D. HIV prevention in gay bathhouses and sex clubs across the United States. J Acquir Immune Defic Syndr. 2010;55(SUPPL. 2):88-90.

46. Woods WJ, Sheon N, Morris JA, Binson D. Gay bathhouse HIV prevention: The use of staff monitoring of patron sexual behavior. Sex Res Soc Policy. 2013;10(2):77-86.

47. Debattista J. Health promotion within a sex on premises venue: notes from the field. Int J STD AIDS. 2015 Dec 28;26(14):1017-21.

48. Krishnan A, Ferro EG, Weikum D, Vagenas P, Lama JR, Sanchez J, et al. Communication technology use and mHealth acceptance among HIV-infected men who have sex with men in Peru: Implications for HIV prevention and treatment. AIDS Care - Psychol Socio-Medical Asp AIDS/HIV. 2015;27(3):27382.

49. Chow JY, Konda KA, Calvo GM, Klausner JD, Caceres CF. Demographics, Behaviors, and Sexual Health Characteristics of High Risk Men Who Have Sex With Men and Transgender Women Who Use Social Media to Meet Sex Partners in Lima, Peru. Sex Transm Dis. 2017;44(3):143-8.

50. Cáceres C, Cueto M, Palomino N. Sexual and Reproductive Rights Policies in Peru: Unveiling False Paradoxes. In: Parker R, Petchesky R, Sember R, editors. SexPolitics - Reports from the Front Lines. Sexuality Policy Watch; 2008. p. 127-66.

51. Maiorana A, Kegeles S, Salazar X, Konda K, Silva-Santisteban A, Cáceres C. 'Proyecto Orgullo', an HIV prevention, empowerment and community mobilisation intervention for gay men and transgender women in Callao/Lima, Peru. Glob Public Health. 2016 Sep 13;11(7-8):1076-92.

52. Garcia J, Amaya ·, Perez-Brumer G, Cabello · Robinson, Clark JL. \&quot;And Then Break the Cliché\&quot;: Understanding and Addressing HIV Vulnerability Through Development of an HIV Prevention Telenovela with Men Who Have Sex with Men and Transwomen in Lima, Peru. Arch Sex Behav. 2018;3:1995-2005.

53. Cáceres CF, Aggleton P, Galea JT. Sexual diversity, social inclusion and HIV/AIDS. AIDS. 2008;22 Suppl 2(Suppl 2):S45.

54. REDCap [Internet]. [cited 2019 Nov 30]. Available from: https://www.project-redcap.org/

55. Long JE, Ulrich A, White E, Dasgupta S, Cabello R, Sanchez H, et al. Characterizing Men Who Have Sex with Transgender Women in Lima, Peru: Sexual Behavior and Partnership Profiles. AIDS Behav. 
2019;(0123456789).

56. OANDA Currency Converter [Internet]. [cited 2018 Nov 26]. Available from: https://www1.oanda.com/currency/converter/

57. Torres TS, Konda KA, Hamid Vega-Ramirez E, Elorreaga OA, Diaz-Sosa D, Hoagland B, et al. Factors associated with willingness to use pre-exposure prophylaxis in Brazil, Mexico, and Peru: Web-based survey among men who have sex with men. J Med Internet Res. 2019;21(6):e13771.

58. Beymer MR, Weiss RE, Bolan RK, Rudy ET, Bourque LB, Rodriguez JP, et al. Sex on demand: Geosocial networking phone apps and risk of sexually transmitted infections among a cross-sectional sample of men who have sex with men in Los Angeles county. Sex Transm Infect. 2014;90(7):567-72.

59. Devost MA, Beymer MR, Weiss RE, Shover CL, Bolan RK. App-Based Sexual Partner Seeking and Sexually Transmitted Infection Outcomes: A Cross-Sectional Study of HIV-Negative Men Who Have Sex with Men Attending a Sexually Transmitted Infection Clinic in Los Angeles, California. Sex Transm Dis. 2018 Jun 1;45(6):394-9.

60. Wang H, Zhang L, Zhou Y, Wang K, Zhang X, Wu J, et al. The use of geosocial networking smartphone applications and the risk of sexually transmitted infections among men who have sex with men: A systematic review and meta-analysis 11 Medical and Health Sciences 1117 Public Health and Health Services. BMC Public Health. 2018 Oct 16;18(1).

\section{Additional File Legend}

Additional File 1 (AdditionalFile1.pdf): Annotated Survey Instrument. Full-length Spanish-language text of survey instrument used in the study, including transitional text and instructional notes used to guide participants, as well as annotations indicating branching logic; all items are in the same order as they appeared to participants taking the actual online survey in REDCap.

\section{Figures}




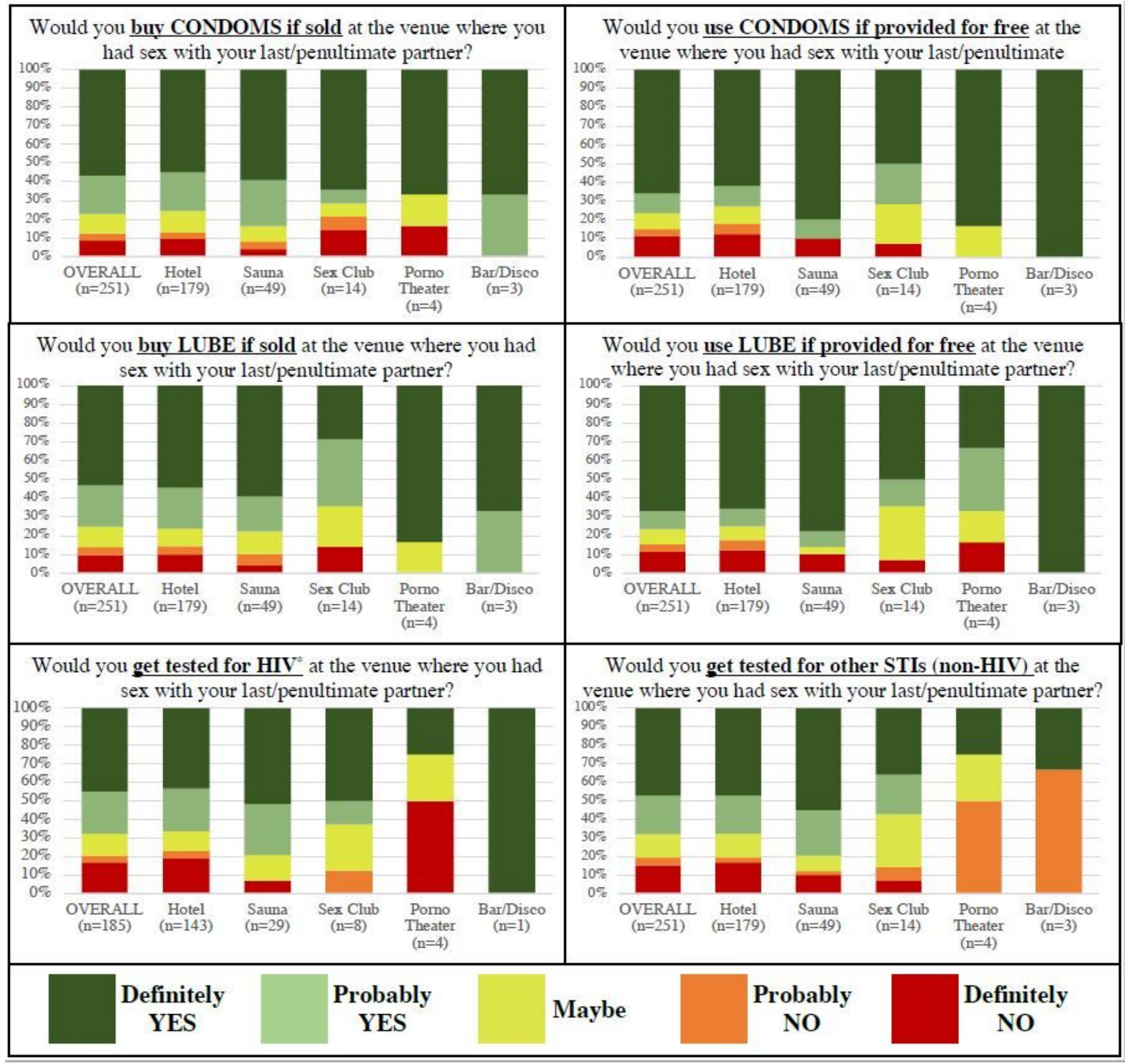

Figure 1

Attitudes Toward Hypothetical SOPV-Based HIV/STI Testing and Sexual Health Interventions. Venue-level data based on an aggregate total of $n=251$ responses corresponding to the SOPV categories where a total of $\mathrm{N}=177$ MSM reported having a sexual encounter with their last $(\mathrm{n}=138)$ and/or penultimate $(n=113)$ partner in the last 3 months. SOPV: sex-on-premise venue; STI: sexually transmitted infection. * Excludes participants who self-report being HIV positive.

\section{Supplementary Files}


This is a list of supplementary files associated with this preprint. Click to download.

- AdditionalFile1.pdf 